\title{
ANTROPOLOGÍA Y SALUD EN COSTA RICA: TRABAJOS FINALES DE GRADUACIÓN DE GRADO Y POSGRADO'
}

\section{ANTHROPOLOGY AND HEALTH IN COSTA RICA: DEGREE AND POSTGRADE FINAL PROJECTS}

\section{Carolina Quesada Cordero*}

\section{RESUMEN}

Este artículo presenta un análisis de los diversos temas, métodos y teorías que se han desarrollado en los Trabajos Finales de Graduación (TFG) sobre salud y enfermedad, realizados en el marco de la Universidad de Costa Rica. El análisis busca aportar a la caracterización de la producción en Antropología médica o de la salud desarrollada en el país. Además, se delimitan una serie de posibles temas para futuros TFG.

PALABRAS CLAVE: COSTA RICA * UNIVERSIDAD * ANTROPOLOGÍA * SALUD * BASE DE DATOS

\section{ABSTRACT}

This article analyses issues, methods and theories developed in the graduate and postgraduate research projects related to health and disease, in the University of Costa Rica. The analysis seeks to contribute to the characterization of the medical Anthropology or the Anthropology health developed in Costa Rica. Also, it shows a series of possible research topics for future projects.

KEYWORDS: COSTA RICA * UNIVERSITY * ANTHROPOLOGY * HEALTH * DATA BASE

$1 \quad$ Este documento se desarrolla en el marco del proyecto de investigación "Causas socioculturales y estructurales del estado de salud sexual y reproductiva en las mujeres de los cantones de Corredores, Golfito y Coto Brus" inscrito en la Vicerrectoría de Investigación de la Universidad de Costa Rica, en el año 2012-2013. Debido a que este proyecto se inscribe dentro de lo que se conoce como Antropología médica o Antropología de la salud, se decidió realizar un análisis de las tesis de grado y posgrado desarrolladas alrededor de dicha temática.

* Escuela de Antropología de la Universidad de Costa Rica (UCR). carolinamqc@gmail.com 


\section{INTRODUCCIÓN}

El presente artículo desarrollará un análisis de los Trabajos Finales de Graduación (TFG) desarrollados desde la Antropología y relacionados con la salud. Los documentos fueron buscados en las bases de datos de la Universidad de Costa Rica, bajo las palabras clave: antropología and médica, antropología and salud, y antropología and embarazo. Los Trabajos Finales de Graduación que no se ubicaron mediante esta búsqueda fueron encontradas en la bibliografía de los documentos previamente identificados. En total, se analizaron 18 TFG, 14 de ellos realizados para optar por el grado de Licenciatura y 4 para optar por el grado de Maestría. Aunque los documentos analizados no siempre se presentan en orden cronológico, si se ofrece un cuadro resumen en el cual se exponen de manera cronológica.

Los TFg analizados trabajan una gran variedad de temas, que van desde la enfermedad, el embarazo y parto, la nutrición, las prácticas médicas populares, la salud sexual y reproductiva, entre otros. Los TFg serán examinados según tres variables: la temática, la metodología y el área teórico-conceptual.

Este documento busca plantear una caracterización inicial sobre el desarrollo de la Antropología médica en Costa Rica, lo cual contribuirá con el avance de esta subdisciplina al identificar los temas estudiados, las perspectivas teóricas que los han enriquecido $y$ las metodologías que se han utilizado $y$ de esta manera, determinar temáticas por explorar, posibles vías de análisis y metodologías exitosas para el desarrollo de estudios en salud.

El artículo ofrece una contextualización inicial en la cual se señalan algunos de los acontecimientos $y$ expositoras más relevantes del desarrollo de la Antropología médica en Costa Rica, así como, una exposición general de los TFg analizados. Seguidamente, se inicia con el análisis temático, en el cual se profundiza en los temas trabajados por los TFG estudiados. Luego, se presenta el análisis metodológico, en el cual se revisan los métodos y técnicas más utilizados. Posteriormente, se presenta el análisis teórico-conceptual en el cual se sistematizan las tendencias teóricas y conceptuales más comunes, así como, la forma en que estas fueron utilizadas. Finalmente, se presenta una serie de conclusiones generales sobre el análisis desarrollado.

\section{CONTEXTUALIZACIÓN}

La Antropología médica o de la salud es una subdisciplina de la Antropología que estudia los fenómenos relacionados con el proceso salud-enfermedad y los sistemas de curación $y$ atención en salud. Esta subdisciplina toma fuerza después de la Segunda Guerra Mundial y se consolida en países como Estados Unidos, México e Inglaterra, durante la segunda mitad del siglo xx (Singer y Baer, 2012; Menéndez, 1990). En Costa Rica, el estudio antropológico de la salud adquiere importancia a partir de la década de 1970, a través de los estudios realizados por María Eugenia Bozzoli² y por Marta Eugenia Pardo ${ }^{3}$.

El tema de la salud-enfermedad, también se vuelve relevante a nivel de Trabajos Finales de Graduación (TFG). Según un estudio realizado por Alpízar, Campos y Zúñiga (2013), entre 1977 y el 2011, se aprobaron un total de 129 TFG para optar por el grado de Licenciatura en Antropología Social, de las cuales un $11 \%$ (14 $\mathrm{TFG}$ ) trabajaron el tema de salud. En el caso de la Maestría en Antropología, del 2001 al 2011, se aprobaron 32 tesis, de las cuales un $12,5 \%$ (4 tesis) abordaron alguna temática relacionada con la salud (Murillo, 2012). Estos estudios se han llevado a cabo a lo largo de

$2 \quad$ María Eugenia Bozzoli consolidó junto a Carlos Aguilar Piedra, la disciplina antropológica en la Universidad de Costa Rica, a partir de la década de los 60 (Ibarra, 2010:164). Durante su carrera escribió varios artículos en los que trabajó el tema de salud, como son: "La Conceptualización y Técnica del Soplo en la Medicina Aborigen Bribri", publicado en 1980 y "La Medicina Aborigen Americana", publicado en 1986. Actualmente, es profesora emérita de la Universidad de Costa Rica.

3 Marta Eugenia Pardo fue profesora catedrática en la Universidad de Costa Rica. Trabajó en esta institución entre 1971 y 1995 . Algunas de sus publicaciones en el tema de salud incluyen: "La Medicina Popular en el Capitalismo Dependiente" (1985) y "Alcohólicos Anónimos: un ejemplo de prestación total" (1986). 
cuatro décadas en el marco de la Escuela de Antropología y Sociología (1974-2009), en la actual Escuela de Antropología (2010-2013) ${ }^{4}$ $y$ en el Posgrado de Antropología (1997-2013), del Sistema de Estudios de Posgrado de la Universidad de Costa Rica.

En el contexto de la Universidad de Costa Rica, los TFG pueden ser desarrollados en cuatro modalidades a nivel de Licenciatura $y$ una modalidad a nivel del Posgrado. Las modalidades existentes en Licenciatura incluyen: a) tesis, la cual, según el "Reglamento de Trabajos Finales de Graduación" (ucr, 1980), consiste en un trabajo de investigación que genera un aporte significativo al tema investigado; b) seminario de graduación, que está a cargo de un profesor(a) que dirige el trabajo y que busca que los y las estudiantes “...mediante su participación reiterada alrededor de algún problema científico o profesional, se familiarizan con las teorías y métodos de investigación propios de la disciplina y su aplicación a casos específicos" (UCR, 1980:2); c) proyecto de graduación, modalidad "...teórico-práctica dirigida al diagnóstico de un problema, su análisis y a la determinación de los medios válidos para resolverlo" (ucR, 1980:2) y d) práctica dirigida, que es una aplicación de conocimiento teórico especializado en una institución pública o privada (ucR, 1980:3). A nivel de Maestría, la única modalidad disponible para estudiantes de Antropología, es la de tesis para Maestría académica, la cual, al igual que la tesis de Licenciatura, busca que se genere un aporte a la disciplina a través de la realización de una investigación. En el caso de Maestría, las tesis deben realizarse de manera individual.

El primer TFG que desarrolla un tema asociado con la salud en la Escuela de Antropología y Sociología, es la práctica dirigida de Giselle Chang y Marlen Castro, "Creencias y actitudes de las primigestas con relación al embarazo, parto y crianza de los niños”, presentada en

4 La Escuela de Antropología y Sociología, con los Departamentos de las respectivas disciplinas, funcionó de 1974, cuando se funda la Facultad de Ciencias Sociales, hasta el año 2009, en que se crean dos unidades académicas distintas: Escuela de Antropología y Escuela de Sociología (Ibarra, 2010:165).
1979, en la cual se investigan las percepciones, creencias $y$ valores asociados al embarazo, parto, puerperio y crianza de los niños y las niñas, por parte de un grupo de mujeres primigestas que asisten a los centros de salud de Santa Ana, Cartago y Heredia. Dicho Tfg utiliza elementos teóricos de la Escuela de Cultural y Personalidad, citando a autoras clásicas como Margaret Mead, Cora DuBois y Ruth Benedict.

El tema de salud toma aún más importancia en la Antropología costarricense en 1984, cuando se publican, en la revista Cuadernos de Antropología, una serie de documentos trabajados durante un seminario-taller sobre Antropología y Salud (Bozzoli, 1984). Dicho seminario-taller se organizó con el objetivo de ofrecer un espacio de reunión e intercambio de experiencias a un pequeño grupo de antropólogos, graduados del Departamento de Antropología, que trabajaban temas relacionados con la Antropología médica, en instituciones públicas (Bozzoli, 1984). Este seminario-taller contó con la participación de Antropólogas de gran trayectoria como María Eugenia Bozzoli, Marta Eugenia Pardo y Carole E. Hill, así como con la participación de Ramiro Barrantes, doctor en genética y población.

A partir de los trabajos presentados durante este seminario-taller, se identificaron problemáticas evidenciadas por el trabajo realizado en Antropología médica en el país (Hill, 1984). Las problemáticas identificadas se relacionan con dos temas:

1) La diferencia en los enfoques utilizados por la biomedicina y las ciencias sociales, que ha repercutido en una falta de comunicación entre profesionales de estas disciplinas. Al respecto se señala que la biomedicina "...ha adoptado un reduccionismo biológico y prestado poca atención a los factores socio-culturales en la salud" (Hill, 1984: 135); a esto se agrega la prevalencia de un enfoque curativo, dejando de lado las acciones a nivel preventivo.

2) Las inequidades existentes en el sistema de atención en salud en Costa Rica, las cuales se evidencias en dos niveles: entre 
los grupos de altos ingresos y los grupos de bajos ingresos, $y$ entre la zona rural $y$ la zona urbana. Al respecto Hill señala: “...la impresión general es que la gente pobre $y$ quienes viven en áreas rurales no están recibiendo un trato igual al de la gente que vive en área urbanas" (1984:136).

Durante las décadas de 1980 y 1990, se presentan ocho TFG $y$ un total de nueve entre el 2001 y el $2011^{5}$. Los documentos estudiados presentan una variedad de temas que incluyen: salud sexual y reproductiva (una tesis de Maestría), salud ocupacional (dos tesis de Licenciatura), análisis de sistemas de atención en salud (una práctica dirigida que analiza el tema a nivel institucional $y$ una tesis de Licenciatura que lo analiza desde la perspectiva de los usuarios), análisis de prácticas alimenticias (dos tesis de Maestría), enfermedades (dos prácticas dirigidas, una sobre leishmaniasis cutánea en Acosta $y$ otra sobre vIH/SIDA, $y$ tres tesis de Licenciatura una sobre hipertensión y diabetes en mujeres, otra sobre leishmaniasis cutánea entre los indígenas cabécares, $y$ la última sobre $\mathrm{VIH} / \mathrm{SIDA}$ ), salud mental (un seminario de graduación), embarazo y parto (una práctica dirigida, un seminario de graduación y dos tesis de Licenciatura) y prácticas curativas populares (una tesis de Maestría).

Diecisiete de los dieciocho TFG analizados, son de carácter cualitativo y utilizan técnicas características del método etnográfico como son la entrevista en profundidad y la observación participante. El TFG restante basa su investigación en el uso de técnicas cuantitativas y del estudio genético. La utilización de recursos teóricos es muy variada, incluyendo referencias a antropólogos clásicos y a teóricos de gran importancia como: Pierre Bourdieu y Michel Foucault. También, es recurrente la utilización de aportes desarrollados por Eduardo Menéndez, cuya trayectoria en la Antropología médica latinoamericana es de gran

$5 \quad$ En total, incluyendo la Práctica Dirigida de Chang y Castro (1979), $y$ las tesis presentadas entre 1980 y el 2011, se encontraron 18 TFG relacionados con el tema de salud. importancia, debido a que ayudó a consolidar la subdisciplina en México, mediante la formación de antropólogos médicos en la Escuela Nacional de Antropología e Historia (enaH), entre 1985 y el 2000 (Campos-Navarro, 2010) y la publicación de obras de gran importancia como: "Poder, estratificación y salud: análisis de las condiciones sociales y económicas de la enfermedad en Yucatán" (1981) y "Antropología médica: orientaciones, desigualdades y transacciones" (1990).

En general, se rescata el tono crítico en los TFG al señalar deficiencias en el sector salud $y$ problemáticas a nivel socio-cultural en relación con la atención en salud, la percepción $y$ prevención de enfermedades. Algunas de las críticas señalan: la existencia de políticas en salud limitadas y deficientes (Kooper, Morera y Orellana, 1987; Brenes, Castro y Pinto, 1988; Beale y Clare, 1989; Gómez y Sánchez, 1991; González y Camacho, 2004; Morales, 2010 y Gutiérrez, 2011), la imposición del sistema médico hegemónico (Kooper, Morera y Orellana, 1987; Beale y Clare, 1989; Ketelhohn, 1996; Palma, 2001; González y Camacho, 2004; Zapparoli, 2004; Solano, 2005; Monestel, 2008; Barrantes y León, 2009), las dificultades observadas en la interacción entre prestadores de servicios de salud $y$ beneficiarios de dichos servicios (Beeche, 1992; Palma, 2001; González y Camacho, 2004; Monestel, 2008), los roles de género establecidos, los cuales recargan el cuido de enfermos en las mujeres (Chang y Castro, 1979; Brenes, Castro y Pinto, 1988; Gómez y Sánchez, 1991; Ketelhohn, 1996; Mojica, 1998; Palma, 2001; González y Camacho, 2004; Zapparoli, 2004; Monestel, 2008 y Gutiérrez, 2011).

A nivel de autoría, un $88 \%$ de los TFG analizados fueron escritos por mujeres. El género de la autora o autor de los documentos toma relevancia ante el señalamiento que hacen Chang y Castro (1979), Brenes, Castro y Pinto (1988), Gómez y Sánchez (1991), Ketelhohn (1996), Mojica (1998), Palma (2001), Zapparolli (2004), González y Camacho (2004), Monestel (2008) y Gutiérrez (2011), en relación con el papel de la mujer en, lo que Zapparolli llama "...el mantenimiento y recuperación de la salud..." 
(2004: 6). Al respecto, esta misma autora afirma que "...es en la mujer en la que recae con mayor fuerza..." el requerimiento de mantener $y$ recuperar la salud, no solo de su familia, sino también de sus vecinos y demás miembros de la comunidad. En relación con esta afirmación, es interesante observar que a nivel investigativo, el tema de la salud también ha sido mayoritariamente abordado por mujeres. Consecuente- mente, las temáticas trabajadas han enfatizado en la realidad que enfrentan las mujeres ante situaciones que requieren asistencia médica, dejando un vacío importante en relación con las situaciones que enfrentan los hombres.

A continuación se presenta el cuadro 1 con la intención de ofrecer una ubicación temporal y temática de los TFG, que faciliten la comprensión del subsiguiente análisis. 


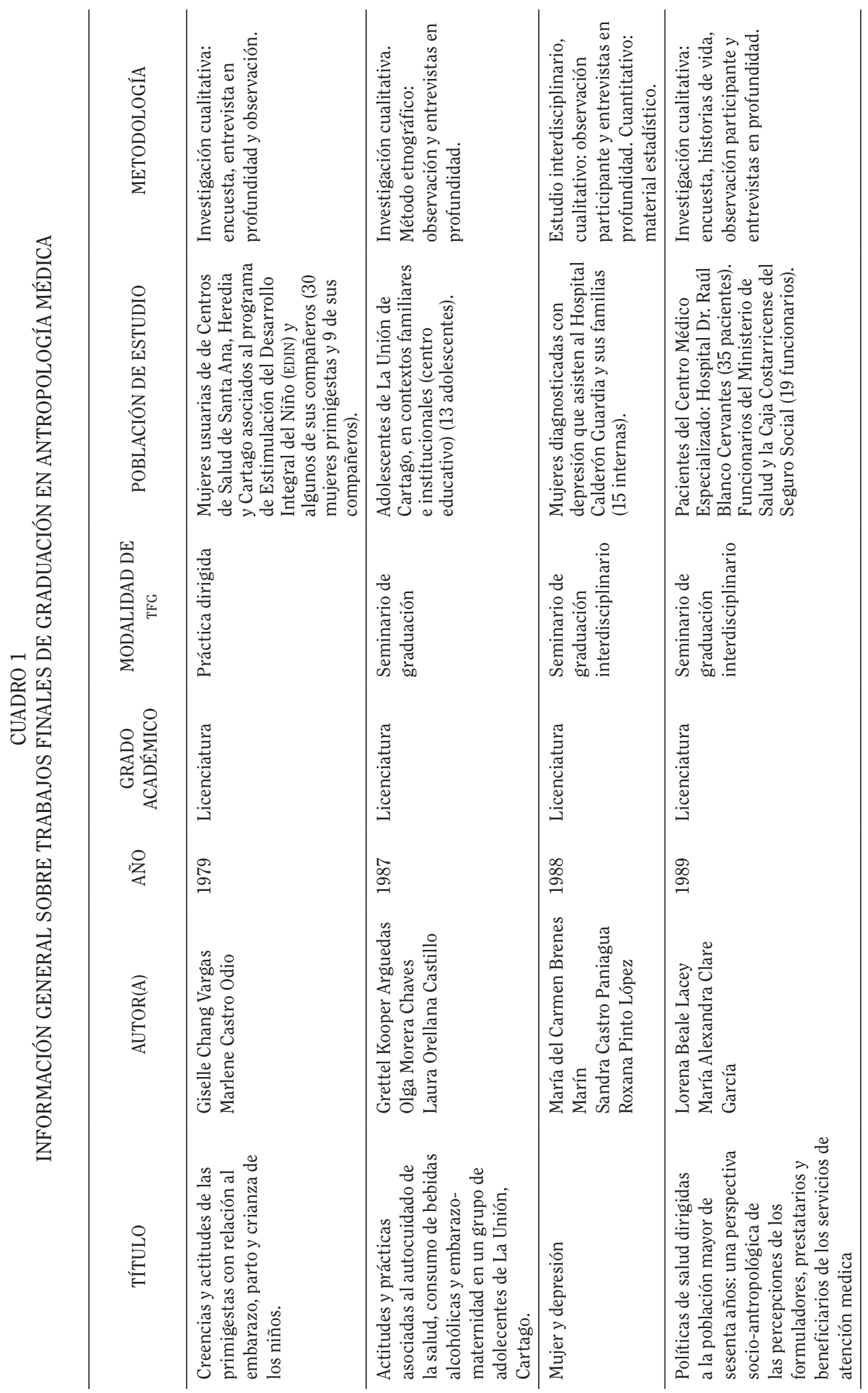

(c) (1) $($ ) Rev. Ciencias Sociales Universidad de Costa Rica, 146: 167-185 / 2014 (IV). (ISSN: 0482-5276) 


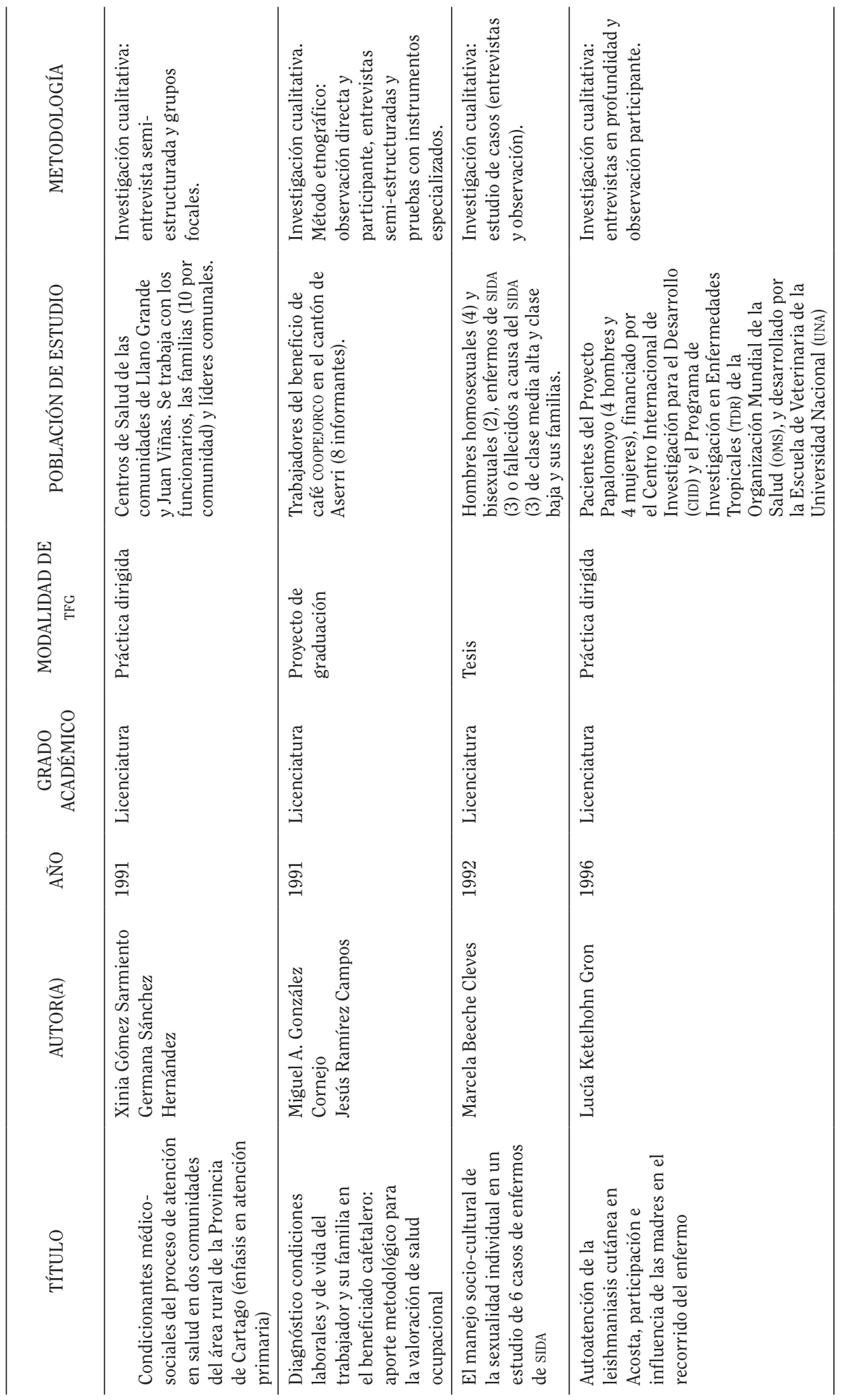




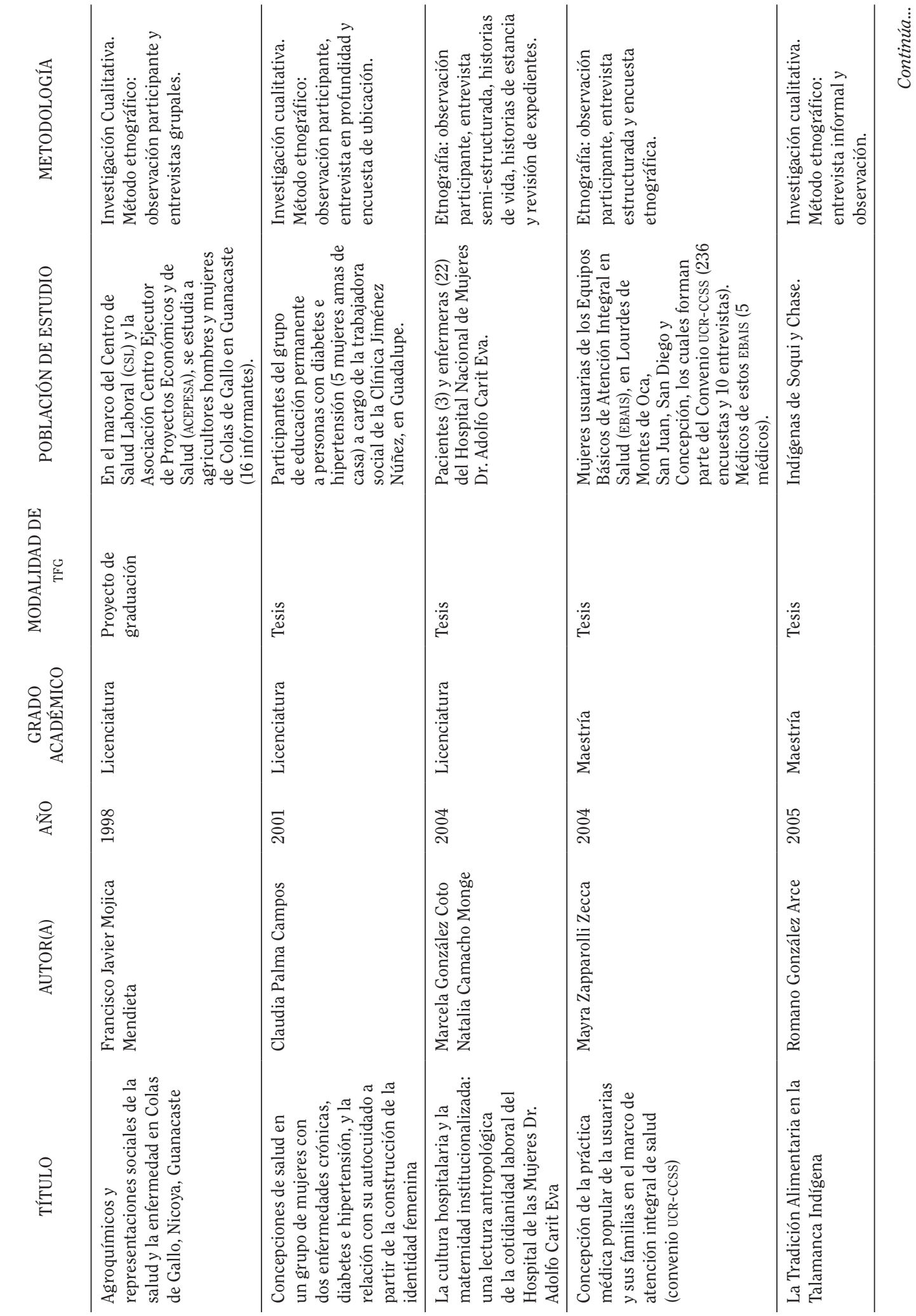




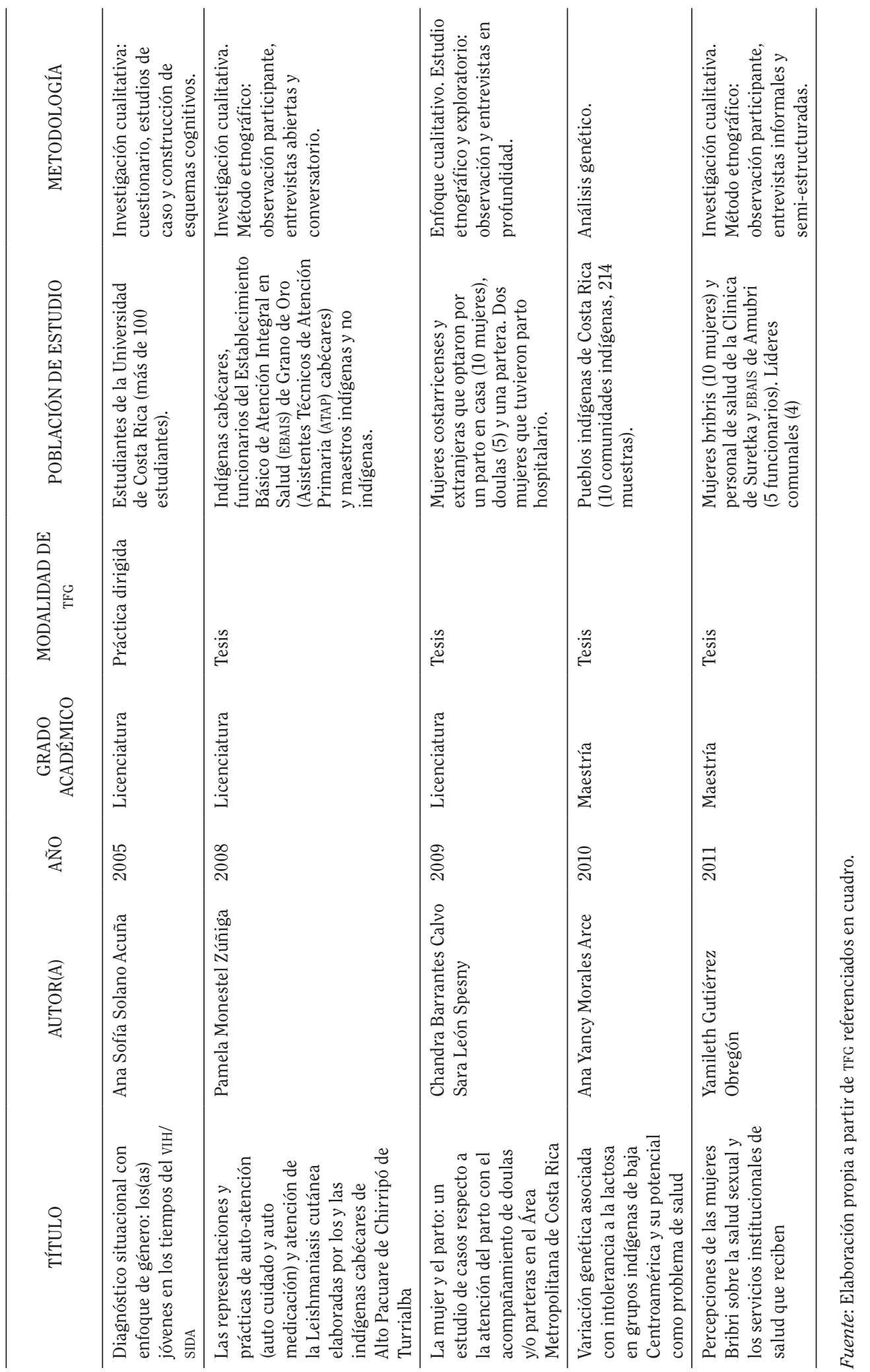


Es importante señalar en este punto, que este estudio no agota el tema de la Antropología médica o de la salud en Costa Rica. Existe un amplio grupo de artículos de revistas que no han sido aún analizados. Dentro de estos artículos se encuentran los aportes de Marta Eugenia Pardo y María Eugenia Bozzoli. Además, se cuenta con una producción desarrollada por antropólogas y antropólogos extranjeros que llevaron a cabo trabajo de campo en el país. Finalmente, es importante considerar la posibilidad de recobrar reseñas y experiencias alrededor de esta temática a través de entrevistas a colegas que hayan trabajado en instituciones dedicadas a investigar los procesos de saludenfermedad y los sistemas de atención.

\section{ANÁLISIS TEMÁTICO}

A lo largo de más tres décadas de TFG, se ha logrado acumular una gran variedad de temas estudiados. Los temas van desde el parto en diferentes contextos, hasta varias enfermedades como la leishmaniasis cutánea y el viH/ SIDA. El gráfico 1 ofrece una orientación cuantitativa sobre la producción temática.

\section{GRÁFICO 1}

TFG EN ANTROPOLOGÍA MÉDICA DE 1979 A 2011, SEGÚN TEMA DESARROLLADO

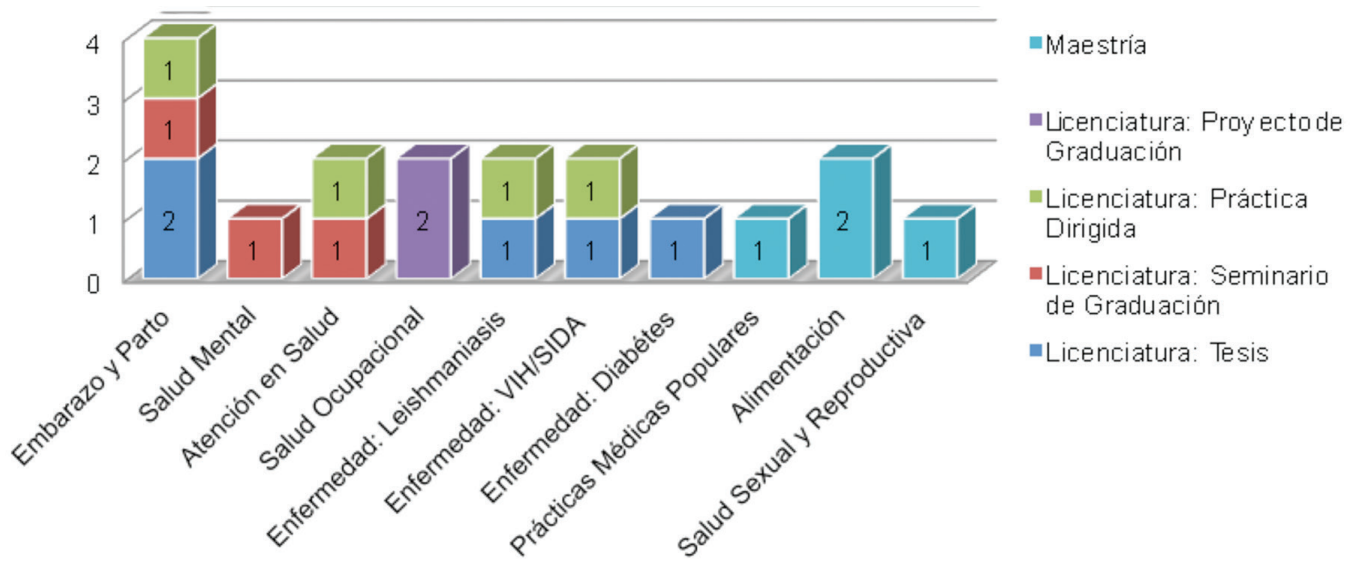

Fuente: Elaboración propia a partir de TFG referenciados en cuadro 1.

A continuación se hace referencia a los temas trabajados y las perspectivas desde las cuales se abordaron:

$\diamond \quad$ Embarazo, parto y post-parto como tema, ha tenido relevancia en dos periodos, a finales de 1970 y durante 1980, y en la primera década del siglo xxı. Este tema ha sido analizado desde diferentes contextos $y$ con mayor o menor énfasis en el proceso parto. El análisis realizado por Chang y Castro (1979), por ejemplo, trabaja el tema no solo desde el proceso de embarazo, parto y post-parto, sino también desde las creencias, valores y percepciones sobre la crianza de los hijos(as), recurriendo a los relatos de infancia $y$ a las expectativas en relación con los hijos(as). Por otro lado, González y Camacho (2004) enfatizan en el proceso de parto institucionalizado, acompañando a las mujeres en labor de parto.

$\diamond \quad$ El trabajo realizado por Brenes, Castro y Pinto (1988) sobre la depresión en mujeres costarricenses es el único TFG sobre salud mental a la fecha. El documento enfatiza en las dificultades que tienen las mujeres para cumplir con 
los requerimientos sociales asignados (cuidado de la familia, en muchos casos, manutención, papel de madre y esposa, entre otros) y las consecuencias de dicha imposición.

$\diamond \quad$ La atención en salud se analiza en dos contextos: desde los servicios ofrecidos a la población mayor de 60 años (tanto a través de políticas públicas, como en el caso concreto del Hospital Dr. Raúl Blanco Cervantes) (Beale y Clare, 1989) y desde los servicios ofrecidos en dos comunidades de Cartago, así como, su impacto desigual (Gómez y Sánchez, 1991).

$\diamond \quad$ El análisis de la salud-enfermedad asociada a las prácticas productivas, se plantea en dos proyectos de graduación. Estos dos documentos son los únicos con autoría masculina y de los pocos TFG analizados que trabaja directamente con hombres. El análisis se desarrolla desde dos contextos distintos: uno en Aserrí, en un beneficio de café (González y Ramírez, 1991) y el otro en Nicoya, con una comunidad de agricultores donde se presentan tanto prácticas de uso de agroquímicos, como prácticas agrícolas orgánicas (Mojica, 1998). En este último documento se trabaja la salud-enfermedad como una representación social, dicha representación no se vincula directamente con el uso de agroquímicos, en muchos casos, porque los efectos solo son percibidos a largo plazo.

$\diamond \quad$ Uno de los temas más importantes, por ser el más trabajado (cinco $\mathrm{TFG}$ ), es el de la enfermedad. Los padecimientos analizados son: el viH/sida (Beeche, 1992 y Solano, 2005), la leishmaniasis cutánea (Ketelhohn, 1996 y Monestel, 2008) e hipertensión y/o diabetes (Palma, 2001). Los trabajos desarrollados presentan gran variación entre sí; sin embargo, es importante señalar que los cinco trg que trabajan este tema, conceptualizan a la enfermedad como un fenómeno biosocial, que se desarrolla en un medio sociocultural particular y cuya atención obedece a percepciones culturales sobre la enfermedad, los elementos bio-socioculturales que los provocan, su proceso de cura y la capacidad (emocional, económica y cultural) que tienen las personas de hacerse cargo de dicho padecimiento.

$\diamond \quad$ Zapparolli (2004) analiza prácticas médicas populares ejercidas por mujeres del Valle Central. A través de este estudio, rescata conocimientos $y$ saberes relacionados con padecimientos específicos y con tratamientos para situaciones particulares como el embarazo. Su estudio también rescata creencias alrededor de prácticas como la de conservar los dientes de leche de niños(as) pequeños(as) o el ombligo de los recién nacidos.

$\diamond \quad$ El tema de la alimentación es trabajado desde dos perspectivas diferentes: uno es un análisis del cambio cultural en una comunidad bribri, en la cual se han ido perdiendo las costumbres asociadas al consumo de alimentos recolectados en el bosque y se han adquirido nuevas prácticas asociadas al consumo de productos procesados que empiezan a encontrarse con mayor frecuencia en la comunidad (González, 2005). El otro trg que aborda el tema de la alimentación, lo hace desde el análisis genético de poblaciones indígenas, para determinar la proporción de la población que es intolerante a la lactasa (Morales, 2010). Este último estudio, aunque es solo de carácter estadístico, ofrece información importante que podría generar el desarrollo de futuros trabajos que analicen el consumo de leche desde un punto de vista cualitativo (sobre todo si se toma en cuenta las políticas públicas asociadas a la promoción del consumo de leche como una fuente nutricional importante).

$\diamond \quad$ Gutiérrez (2011) desarrolla un análisis de salud sexual y reproductiva de mujeres bribris en su interacción con los servicios de salud de la Caja Costarricense del Seguro Social (ccss) presentes en la zona. En este documento se trabaja la salud sexual y reproductiva desde un enfoque 
de interculturalidad y promoción de la salud, en el cual las mujeres sean respetadas en cuanto a sus prácticas culturales asociadas a la salud, pero además, se genere un diálogo que permita la comprensión y el conocimiento mutuo, de manera que los procesos de promoción sean más efectivos.

\section{ANÁLISIS METODOLÓGICO}

A nivel metodológico, no se percibe mucha variación. En general, prevalece la investigación cualitativa (17 de 18 TFG utilizan metodología cualitativa) ${ }^{6}$ como eje central del planteamiento metodológico. En algunos casos se hace mención a la etnografía como el método seleccionado (Kooper, Morera y Orellana, 1987; González y Ramírez, 1991; Mojica, 1998; González y Camacho, 2004; Zapparolli, 2004; Monestel, 2008 y Gutiérrez, 2011). Otros documentos hablan de estudios exploratorios (Chang y Castro, 1979; Brenes, Castro y Pinto, 1988; Gómez y Sánchez, 1991; Palma, 2001; Zapparolli, 2004; Barrantes y León, 2009) o interdisciplinarios (Brenes, Castro y Pinto, 1988; Beale y Clare, 1989). En dos casos se plantean los estudios de caso como el elemento que guiará el desarrollo de la investigación (Beeche, 1992 y Solano, 2005).

La observación participante, característica del método etnográfico, fue utilizada en trece TFG $y$ la entrevista (ya sea en profundidad, semi-estructurada, informal o abierta) en dieciséis de los documentos analizados. Estas dos técnicas son las más utilizadas para la recopilación de datos cualitativos y por lo general, se acompañan de otras técnicas para recopilar algunos datos cualitativos sobre experiencias o percepciones particulares $y$ datos cuantitativos. Algunas de las otras técnicas utilizadas son:

6 Con excepción de los trabajos de Brenes, Castro y Pinto (1988), González y Ramírez (1991), Zapparolli (2004) y Morales (2010), que no hacen referencia a la investigación cualitativa dentro del planteamiento metodológico. No obstante, Brenes, Castro y Pinto (1988) hacen referencia al uso de técnicas de investigación cualitativas, mientras que González y Ramírez (1991) y Zapparolli (2004), afirman que su estudio es etnográfico. investigación bibliográfica (en algunos casos con un peso importante, como en la tesis de González, 2005), la encuesta o cuestionario, grupos focales, inventario вем (para identificar la conceptualización de los roles de género), historia de vida, historia de estancia, revisión de documentos y expedientes, diario o notas de campo, construcción de esquemas cognitivos, talleres, relatos, fotografías y reuniones.

Aunque los TFG analizados fueron desarrollados en diferentes modalidades, no se encuentran grandes diferencias entre los documentos. En general, estos tienen una estructura similar, en la cual se plantean objetivos, propuestas teórico-conceptuales, una propuesta metodológica y luego se exponen los resultados. Las prácticas dirigidas, aunque se realizaron dentro de una institución, presentan, al igual que los otros trabajos, gran cantidad de datos sobre la realidad en la cual se trabajó.

\section{ANÁLISIS TEÓRICO-CONCEPTUAL}

Los TFg analizados presentan gran variedad en las propuestas teórico-conceptuales retomadas, así como en la extensión e importancia que tienen estas para la investigación. No obstante, se pueden identificar algunas generalidades importantes $y$ señalar los casos que sobresalen, debido a la propuesta teórica utilizada, como por el peso que esta adquiere a lo largo de los documentos.

En principio, es importante señalar que todas las propuestas teórico-conceptuales analizadas, coinciden en que la salud y los demás fenómenos relacionados (enfermedad, parto, adolescencia, envejecimiento y nutrición), no son solo fenómenos biológicos, sino también culturales y sociales. Por lo tanto, las características culturales, sociales, políticas, económicas y organizacionales, son ejes centrales en las propuestas teórico-conceptuales.

A nivel histórico, se identificaron tres tendencias marcadas, las cuales se caracterizan por la utilización persistente de una propuesta teórica o conceptual durante un periodo de tiempo; sin embargo, que una propuesta adquiera relevancia por su reiterado uso en un periodo particular, no quiere decir que no se siga 
utilizando en otros periodos. Lo que es evidente, es que no se utilizan con la misma centralidad e importancia. A continuación se señalan estas tendencias:

1) Los Tfg realizados a finales de la década de 1970 y durante la década de $1980^{7}$, hacen referencia a la Escuela de Cultura y Personalidad (citando sobre todo a Mead y Benedict). Dentro de este marco teórico, utilizan la noción de "procesos de socialización", para hacer referencia al proceso de adquisición de la cultura. Este tema tiene especial relevancia en los TFG de Chang y Castro (1979), y Kooper, Morera y Orellana (1987), debido a que estos documentos trabajan alrededor del periodo de crianza, en el cual se educa a nuevos individuos en una cultural particular. En el caso de Brenes, Castro y Pinto (1988), la noción de "procesos de socialización" es relevante, por ofrecer una explicación a la adquisición de normas asociadas con el género.

2) Durante la década de 1990, se utilizan mayoritariamente propuestas asociadas a las relaciones de clase $y$ poder. No es casualidad que durante este periodo se realicen los únicos dos trabajos relacionados con la Salud Ocupacional, haciendo referencia a las condiciones de los trabajadores $y$ a las posibilidades que estos tienen como agentes de modificación de las prácticas laborales riesgosas. Dentro de este marco teórico, se hace referencia a la importancia de las condiciones socio-económicas de esos trabajadores, así como, elementos que influyen en su salud.

3) El concepto de poder se trabaja desde la perspectiva foucaultiana, es decir, el poder se considera un elemento que existe cuando se ejerce $y$ no se puede poseer o acumular. El ejercicio del poder no se limita a ciertos grupos dentro de la organización sociopolítica, ya que puede ser

$\overline{7} \quad$ El TFG de Beale y Clare (1989) no presenta estas características. Esto puede estar asociado al tema desarrollado, el cual no está directamente relacionado con procesos de socialización y género. ejercida desde distintas posiciones socioculturales (Beeche, 1992; Ketelhohn, 1996 y Mojica, 1998). La propuesta de Ketelhohn (1996), por ejemplo, utiliza el concepto de poder desarrollado por Foucault para analizar las prácticas de autoatención de la leishmaniasis cutánea en Acosta. De acuerdo con esta autora, la autoatención representa el espacio cultural en el cual las mujeres logran ejercer el poder ${ }^{8}$ al tomar decisiones sobre el diagnóstico y atención de este padecimiento. También está bajo el control de las mujeres, la decisión de renunciar a este poder $y$ asistir al centro médico.

4) La tercera tendencia en cuanto a propuestas teórico-conceptuales de los TFG, se encontró en el concepto de cuerpo, el cual adquiere relevancia a partir del siglo $\mathrm{xxI}^{9}$. La centralidad del cuerpo se observa con mayor claridad en los trabajos desarrollados por González y Camacho (2004), y Barrantes y León (2009), quienes trabajan el tema del parto en dos contextos distintos (institucionalizado y no institucionalizado, respectivamente). La insistencia en lo corporal encuentra sentido al ser el parto un proceso de cambio acelerado del cuerpo, con efectos importantes en las sensaciones y percepciones del mismo. Además, el cuerpo, es entendido como un mediador social, entre el ser humano y el mundo social $y$ cultural, por lo tanto, es a partir de las percepciones que se tienen de nuestro cuerpo, que se aborda ese espacio (Barrantes y León, 2009). González y Camacho (2004) hacen referencia al control sobre el cuerpo que se promueve en Occidente, particularmente, en la atención institucionalizada de la salud y en este caso, del parto.

$8 \quad$ En esta misma línea, Zapparolli (2004) enfatiza en el control del saber y el conocimiento como un elemento de poder.

9 Este concepto es señalado en todos los TFG desarrollados en este periodo, con excepción de los trabajos de González (2005) y Morales (2010). 
Existen también una serie de elementos teórico-conceptuales que se desarrollan de manera persistente a lo largo de las tres décadas. Las persistencias más sobresalientes son:

1) La perspectiva de género es utilizada en una gran cantidad de TFG; no obstante, es claro que las tendencias de esta perspectiva varían a lo largo del tiempo. A finales de 1970 y durante 1980, la perspectiva de género estaba particularmente asociada a los roles de género. Durante la década de 1990, las tesis de Ketelhohn (1996) y Mojica (1998), trabajan el género desde las relaciones de poder y asociado a otras características como la clase social. Durante la primera década del siglo xxi, se siguen trabajando ambas tendencias (la de roles de género y relaciones de poder), mientras se identifican espacios en los cuales se desarrollan las mujeres (en contextos de enfermedad y sistemas de atención (Palma, 2001), como portadoras y reproductoras de la medicina tradicional (Zapparolli, 2004 y Monestel, 2008), y como actoras en procesos de parto (González y Camacho, 2004; Barrantes y León, 2009) y atención en salud (Gutiérrez, 2011)) y se analizan sus experiencias en diversos contextos organizacionales, étnicos y de pobreza.

2) La diferenciación de Modelos Médicos se retoma en varios de los TFG. En general, se reconocen al menos dos modelos médicos, uno asociado al biologismo $y$ al cientificismo, en relación directa con la biomedicina $y$ asociado a una atención individualizada de la salud; $y$ otro asociado a la concepción global de los padecimientos y al pragmatismo. El primer modelo se denomina Modelo Médico Hegemónico (Kooper, Morera y Orellana, 1987; Beale y Clare, 1989) o Práctica Médica Institucional (Zapparolli, 2004); esta misma idea es desarrollada en otros tFg pero sin un término específico (Gómez y Sánchez, 1991; Palma, 2001; González y Camacho, 2004; Monestel, 2008). El segundo modelo se ha denominado Modelo Médico Popular
(Kooper, Morera y Orellana, 1987) o Modelo Médico Alternativo (Beale y Clare, 1989) o Prácticas Curativas Populares (Zapparolli, 2004). En el caso de Beale y Clare, se hace referencia a un tercer modelo, denominado Modelo Médico de Autoatención, el cual se considera el primer nivel de atención, en el que se generan prácticas de autoprescripción y automedicación; estas prácticas son características del núcleo familiar. Este tercer modelo también ha sido incorporado en otras propuestas teórico-conceptuales de los TFG analizados, haciendo referencia a la automedicación como un patrón cultural (Kooper, Morera y Orellana, 1987) y a la autoatención como "... no solo la forma de atención más constante, sino el principal núcleo de articulación práctica de las diferentes formas de atención" (Monestel, 2008:114). Es importante rescatar que un grupo importante de autoras señalan la autoatención como un elemento asociado al género femenino (Chang y Castro, 1979; Brenes, Castro y Pinto, 1988; Ketelhohn, 1996; Mojica, 1998; Palma, 2001; Zapparolli, 2004 y Monestel, 2008).

3) La noción de "proceso salud-enfermedad" es entendida como aquellos elementos que provocan el desarrollo de enfermedades. Para explicar la aparición de enfermedades se han generado varias propuestas. Una de ellas habla de tres corrientes: la corriente biológica, en la cual los aspectos biológicos son los que desencadenan la enfermedad; la ecológica, en esta corriente la enfermedad se desarrolla a partir de la interacción entre los seres humanos, así como entre estos y el medio ambiente; $y$ la sociohistórica, en la cual la enfermedad se analiza en sociedades concretas, desde una perspectiva bio-social y colectiva (Beale y Clare, 1989). También se hace referencia a tres modelos de explicación de la enfermedad, la salud y los accidentes laborales en la tesis de González y Ramírez (1991): Modelo Monocausal, 
donde un único agente es causante de la enfermedad y la salud se ve como un proceso individual; Modelo Multicausal, el cual toma en cuenta las características socioeconómicas y culturales a la hora de establecer la causa de la enfermedad; $y$ el Modelo Estructural, que establece que la salud y la enfermedad están determinadas por la forma de organización social en términos económicos y sociales (clase, etnia, género, entre otros).

4) En general, los TFg se apegan al Modelo Multicausal o Estructural. La enfermedad es entendida como un proceso que puede ser biológico o social, cuyo desarrollo está determinado por condiciones sociales, económicas, culturales y políticas. Las condiciones de desigualdad se ven enfatizadas en algunos de los documentos analizados, los cuales plantean críticas a nivel estructural, incluyendo tanto las diferencias de clase y género (Brenes, Castro y Pinto, 1988; González y Ramírez, 1991; Ketelhohn, 1996; Mojica, 1998 y Palma, 2001), como la estigmatización que generan enfermedades como el vir/sIDA (Beeche, 1992) o las problemáticas asociadas a las diferencias culturales y los servicios de atención en salud (Gómez y Sánchez, 1991; Monestel, 2008).

5) El análisis de representaciones sociales fue utilizado como teoría en varios estudios; no obstante, solo en dos casos se plantea esta propuesta teórica como elemento clave en el análisis de la realidad (Mojica, 1998 y Monestel, 2008). Las representaciones sociales son elaboraciones dinámicas y no armónicas, asociadas a comportamientos, a la comunicación, a imágenes, significados y sistemas de referencia. A través del análisis de las representaciones sociales es posible identificar las ideas y significados asociados a experiencias de la vida cotidiana en diferentes contextos. Este marco teórico genera aportes importantes para los TFG señalados, al permitirles analizar la salud como una representación social, construida, dinámica y no armónica.
Finalmente, se destacan algunas propuestas particulares, desarrolladas alrededor de temas relevantes:

1) Los patrones alimenticios son acciones realizadas para satisfacer la necesidad de comer. Estos patrones satisfacen otras necesidades a nivel emocional, social y ritual. Los patrones alimenticios tienen tres componentes que son: obtención, preparación y consumo de los alimentos. Además, se mantienen en la medida en que el conocimiento asociado a los mismos se transmite (González, 2005).

2) La co-evolución gen-cultura es una hipótesis que explica porqué algunos grupos de personas presentan tolerancia a la lactasa y otros no. A esta hipótesis también se le llama hipótesis histórico-cultural o de selección natural mediada por la cultura. La hipótesis establece que la tolerancia a la lactosa en algunos grupos, es una adaptación al pastoreo y al consumo de leche (Morales, 2010).

3) En su tesis de maestría, Gutiérrez (2011) hace referencia a la salud sexual y reproductiva desde una perspectiva de derechos, señalando la importancia de defender estos derechos y asegurar la toma libre de decisiones entre las personas, sin importar su bagaje cultural.

4) Finalmente, la enfermería transcultural hace referencia a una propuesta de aplicación de la disciplina a contextos culturales diversos, donde se facilitan herramientas que permitan a los(as) enfermeros(as) decidir sobre la adopción de posibles acciones que resulten coherentes en los diversos contextos culturales (Gutiérrez, 2011).

\section{CONCLUSIONES GENERALES}

El análisis desarrollado permite elaborar una serie de conclusiones sobre el desarrollo de la Antropología médica o Antropología de la salud en Costa Rica. En general, se puede plantear que existen diferencias importantes: 
$\diamond \quad$ La mayoría de los textos desarrolla análisis críticos de la realidad bajo estudio. Señalando de manera intuitiva o explicita elementos estructurales que limitan la capacidad de las personas de mejorar su salud $y$ por lo tanto, su calidad de vida. Algunos de los elementos señalados como limitantes para mejorar la salud son: las particularidades culturales, el género, la clase, la procedencia rural/ urbana y la etnia.

$\diamond \quad$ El género es un elemento que ha sido tomado en cuenta $y$ en el cual se ha insistido al momento de plantear los análisis (al menos en la mayoría de los casos). Pero es importante recordar que el ser mujer puede tener repercusiones distintas según sea la clase, la etnia o la procedencia de la mujer. Un grupo importante de los estudios ponen énfasis en al menos una de estas variables; en los casos en que no fue así, el estudio se habría enriquecido de haberse ahondado en estas.

$\diamond \quad$ El tema del cuerpo toma relevancia en los TFG a partir del siglo XXI. Este elemento no es tan importante en los estudios previos con excepción del trabajo realizado por Brenes, Castro y Pinto (1988), en el cual el cuerpo toma relevancia a nivel de la medicina tradicional. En los trabajos realizados a partir del 2001, el cuerpo es un eje central en el desarrollo de estudios relacionados con: la salud, la enfermedad, los procesos curativos, los procesos reproductivos (desde la gestación - sin importar el método que se utilice para lograrla, sea natural o a través de alguna tecnología- hasta el parto), los servicios en salud, entre otros. basa en los determinantes políticos, ideológicos, culturales, económicos e institucionales de las situaciones de salud de las personas. Al realizar este tipo de acercamiento, es posible alejarse de una visión basada en "los estilos de vida saludables", los cuales son culpabilizantes, al recargar la responsabilidad

en las y los actores, ignorando por lo tanto, aquellos elementos que configuran el contexto en el que se desarrolla la persona y sobre los cuales esta tiene pocas posibilidades de incidir. Al respecto, Menéndez asegura, refiriéndose a la producción en Antropología médica en México, que "la producción antropológica en Latinoamérica, salvo excepciones, permanece inclusive al margen de las corrientes teóricas críticas desarrolladas a nivel internacional, que justamente emergen como alternativas..." (1990: 14). En el caso de Costa Rica, aunque en algunos TFG no se utiliza la producción internacional en Antropología médica, la mayoría de estos documentos logra desarrollar propuestas que se asocian con un marco teórico crítico, el cual hace referencia al estudio de la salud, enfermedad, sistemas de curación, procesos de reproducción, entre otras, tomando en cuenta las "... construcciones culturales dominantes en salud..." (Singer y Baer, 2012: 39). Los TFG que utilizan la producción internacional en Antropología médica recurren a gran variedad de autores en América Latina (como Menéndez), en Estados Unidos (como Mead o Martin) y en Europa (como Devereux).

$\diamond \quad$ Los TFG que no utilizan la literatura internacional en Antropología médica habrían logrado profundizar en algunos elementos relevantes que solo señalan de manera general, ofreciendo un análisis más crítico sobre las realidades estudiadas.

$\diamond \quad$ La mitad de los TFG fueron desarrollados en comunidades rurales del país, de los cuales 3 de estos trabajos fueron elaborados en comunidades indígenas. En general, la proporción es alta; no obstante, es importante seguir promoviendo este tipo de trabajos, de manera que las comunidades con mayores situaciones de vulnerabilidad en el país se sigan viendo beneficiadas de este tipo de estudios.

$\diamond \quad$ Como punto final, es importante señalar que todos los TFG, sin excepción, generaron alguna reflexión en cuanto a posibles 
intervenciones para mejorar la salud $y$ por lo tanto, la calidad de vida de las personas. Estas propuestas de intervención se plantearon a nivel de comunidades, empresas y de los sistemas de atención en salud.

El presente análisis permitió identificar posibles temas de investigación; algunos de los cuales han sido planteados en los TFG como temas que no pudieron ser explorados por falta de tiempo o recursos, otros son temas que surgen luego de revisar y reflexionar sobre estos documentos:

$\diamond \quad$ Mujer y depresión en zonas rurales del país.

$\diamond \quad$ Construcción y vivencia de la masculinidad, en relación con los riesgos de trabajo.

$\diamond \quad$ Cambio cultural en relación con: el sistema de salud costarricense, utilización de prácticas curativas populares, experiencias institucionales de parto, sistemas de autoatención intrafamiliares, entre otros.

$\diamond$ Aprendizaje del reconocimiento de especies botánicas entre los bribris en Talamanca, con el objetivo de recuperar el consumo de quelites (hojas y tallos tiernos de plantas comestibles) en esta comunidad (González, 2005).

$\diamond \quad$ Estudio del impacto sociocultural de la atención en salud-enfermedad de un sistema médico ajeno al autóctono (Monestel, 2008).

$\diamond \quad$ Métodos de planificación familiar en población indígena, utilización de método naturales, así como, la utilización y aceptación de los métodos que ofrece la biomedicina (Monestel, 2008).

$\diamond \quad$ Parto en la cosmovisión indígena (Monestel, 2008).

$\diamond \quad$ Percepciones $y$ creencias de los hombres sobre el proceso de embarazo y parto (Barrantes y León, 2009).

$\diamond \quad$ Violencia de género en población indígena (Gutiérrez, 2011).

$\diamond \quad$ Salud, enfermedad, atención y curación entre los hombres.
Como se mencionó al principio de esta exposición, el trabajo de investigación en salud ha sido desarrollado principalmente por mujeres y sobre mujeres, dejando de lado la visión masculina, no solo con respecto al parto, la enfermedad de las personas que los rodean y el cuido de su familia y comunidad, sino también en relación con su propia salud, las prácticas y significados asociados a su autocuidado, y lo que significa estar enfermo desde una masculinidad construida en una sociedad patriarcal.

\section{BIBLIOGRAFÍA}

LIBROS

Alpízar, Natasha; Campos, Pamela y Zúñiga, Mario. Registro de tesis en el área de Antropología Social producidas por estudiantes del Departamento/Escuela de Antropología de la Universidad de Costa Rica (1977-2011). San José, Costa Rica. Enero, 2013.

Singer, Merril y Baer, Hans. Introducing medical anthropology: a discipline in action. 2da. edición. EeuU: Altamira Press, 2012.

\section{PUBLICACIONES PERIÓDICAS}

Bozzoli, María. "La investigación en Antropología médica en Costa Rica". Cuadernos de Antropología 2. San José, Costa Rica. Universidad de Costa Rica (UCR), 1984: 1-9.

Campos-Navarro, Roberto. "La enseñanza de la Antropología médica y la salud intercultural en México: del indigenismo culturalista del siglo $\mathrm{xx}$ a la interculturalidad en salud del siglo xxI”. Revista Peruana de Medicina Experimental y Salud Pública 27 (1). Lima, Perú. Instituto Nacional de Salud, 2010: 114-122.

Hill, Carole. "Reflexiones sobre el Seminario Taller". Antropología y Salud 3. 1984: 135-138.

Ibarra, Eugenia. "La Antropología Social y la Arqueología en la Facultad de Ciencias Sociales de la Universidad de Costa Rica 
(1967-2009)". Revista Reflexiones 89 (1). San José, Costa Rica. Universidad de Costa Rica (UCR), 2010: 163-173.

\section{TESIS NO PUBLICADAS}

Barrantes, Chandra y León, Sara. "La mujer y el parto: un estudio de casos respecto a la atención del parto con el acompañamiento de doulas $y / 0$ parteras en el Área Metropolitana de Costa Rica”. [Tesis de Licenciatura en Antropología Social]. San José, Costa Rica. Universidad de Costa Rica (UCR), 2009.

Beale, Lorena y Clare, María Alexandra. "Políticas de salud dirigidas a la población mayor de sesenta años: una perspectiva socio-antropológica de las percepciones de los formuladores, prestatarios y beneficiarios de los servicios de atención medica". [Seminario de Graduación de Licenciatura en Antropología Social y Sociología]. San José, Costa Rica. Universidad de Costa Rica (UCR), 1989.

Beeche, Marcela. "El manejo socio-cultural de la sexualidad individual en un estudio de 6 casos de enfermos de SIDA". [Tesis de Licenciatura en Antropología Social]. San José, Costa Rica. Universidad de Costa Rica (UCR), 1992.

Brenes, María del Carmen; Castro, Sandra y Pinto, Roxana. "Mujer y depresión". [Seminario de Graduación de Licenciatura en Antropología]. San José, Costa Rica. Universidad de Costa Rica (UCR), 1988.

Chang, Giselle y Castro, Marlene. "Creencias $y$ actitudes de las primigestas con relación al embarazo, parto y crianza de los niños". [Práctica Dirigida de Licenciatura en Antropología Social]. San José, Costa Rica. Universidad de Costa Rica (UCR), 1979.

Gómez, Xinia y Sánchez, Germana. "Condicionantes médico-sociales del proceso de atención en salud en dos comunidades del área rural de la Provincia de Cartago (énfasis en atención primaria)". [Práctica Dirigida de Licenciatura en Antropología Social].
San José, Costa Rica. Universidad de Costa Rica (UCR), 1991.

Gonzalez, Miguel A. y Ramírez, Jesús. "Diagnóstico condiciones laborales $y$ de vida del trabajador y su familia en el beneficiado cafetalero: aporte metodológico para la valoración de salud ocupacional". [Proyecto de Graduación de Licenciatura en Antropología Social]. San José, Costa Rica. Universidad de Costa Rica (UCR), 1991.

González, Marcela y Camacho, Natalia. "La cultura hospitalaria y la maternidad institucionalizada: una lectura antropológica de la cotidianidad laboral del Hospital de las Mujeres Dr. Adolfo Carit Eva". [Tesis de Licenciatura en Antropología Social]. San José, Costa Rica. Universidad de Costa Rica (UCR), 2004.

González, Romano. "La tradición alimentaria en la Talamanca indígena". [Tesis de Maestría en Antropología Social]. San José, Costa Rica. Universidad de Costa Rica (UCR), 2005.

Gutiérrez, Yamileth. "Percepciones de las mujeres Bribri sobre la salud sexual y los Servicios Institucionales De Salud Que Reciben". [Tesis de Maestría en Antropología Social]. San José, Costa Rica. Universidad de Costa Rica (UCR), 2011.

Ketelhohn, Lucía. "Autoatención de la Leishmaniasis Cutánea en Acosta, participación e influencia de las madres en el recorrido del enfermo". [Práctica Dirigida de Licenciatura en Antropología Social]. San José, Costa Rica.Universidad de Costa Rica (UCR), 1996.

Kooper, Grettel; Morera, Olga y Orellana, Laura. "Actitudes y prácticas asociadas al autocuidado de la salud, consumo de bebidas alcohólicas y embarazo-maternidad en un grupo de adolecentes de La Unión, Cartago”. [Seminario de Graduación de Licenciatura en Antropología Social]. San José, Costa Rica.Universidad de Costa Rica (UCR), 1987. 
Mojica, Francisco Javier. "Agroquímicos y representaciones sociales de la salud $y$ la enfermedad en colas de gallo, Nicoya, Guanacaste". [Proyecto de Graduación de Licenciatura en Antropología Social]. San José, Costa Rica. Universidad de Costa Rica (UCR), 1998.

Monestel, Pamela. "Las representaciones y prácticas de auto-atención (auto cuidado $y$ auto medicación) y atención de la Leishmaniasis cutánea elaboradas por los y las indígenas cabécares de Alto Pacuare de Chirripó de Turrialba". [Tesis de Licenciatura en Antropología Social]. San José, Costa Rica.Universidad de Costa Rica (UCR), 2008.

Morales, Ana Yancy. "Variación genética asociada con intolerancia a la lactosa en grupos indígenas de baja Centroamérica y su potencial como problema de salud". [Tesis de Maestría en Antropología Biológica]. San José, Costa Rica. Universidad de Costa Rica (UCR), 2010.

Murillo, Carmen. Tesis Presentadas en la Maestría Académica en Antropología. Base de datos del Posgrado en Antropología. Inédito. San José, Costa Rica: Universidad de Costa Rica (UCR), 2012.

Palma, Claudia. "Concepciones de salud en un grupo de mujeres con dos enfermedades crónicas, diabetes e hipertensión, y la relación con su autocuidado a partir de la construcción de la identidad femenina". [Tesis de Licenciatura en
Antropología Social]. Universidad de Costa Rica (UCR), 2001.

Solano, Ana Sofía. "Diagnóstico situacional con enfoque de género: los(as) jóvenes en los tiempos del VIH/SIDA". [Practica Dirigida de Licenciatura en Antropología Social]. San José, Costa Rica. Universidad de Costa Rica, (UCR), 2005.

Zapparolli, Mayra. "Concepción de la práctica médica popular de las usuarias y sus familias en el marco de atención integral de salud (convenio UCR-CCSS)". [Tesis de Maestría en Antropología Social] San José, Costa Rica. Universidad de Costa Rica (UCR), 2004.

\section{TEXTOS ELECTRÓNICOS}

Universidad de Costa Rica (UCR). Reglamento de Trabajos Finales de Graduación. 1980. En: <http://www.cu.ucr.ac.cr/normativ/ trabajos_finales_graduacion.pdf $>$ [consultado el 5 de octubre de 2013].

OTROS

Menéndez, Eduardo. Antropología médica: orientaciones, desigualdades y transacciones. México DF, México: Centro de Investigaciones y Estudios Superiores en Antropología Social (CIESAS) y Cuadernos de la Casa Chata, 1990.

Fecha de ingreso: 21/12/2013 Fecha de aprobación: 30/04/2014 
